\title{
Lessons For Human Holobiont Medicine in The Era of SARS-Cov-2
}

\author{
Rodney R Dietert* \\ Department of Microbiology and Immunology, Cornell University, USA \\ *Corresponding author: Rodney R Dietert, Professor Emeritus of Immunotoxicology, Cornell University, USA. \\ To Cite This Article: Rodney R Dietert. Lessons For Human Holobiont Medicine in The Era of SARS-Cov-2. Am J Biomed Sci \& Res. 2021 - 13(2). \\ AJBSR.MS.ID.001849. DOI: 10.34297/AJBSR.2021.13.001849.
}

Received: 眥 June 02, 2021; Published: 眥 June 14, 2021

\begin{abstract}
The current SARS-CoV-2 (Covid 19) pandemic has been tragic but also instructional. It revealed the flaws in life-course health management that caused aging populations to lack resiliency, carry multiple inflammation-driven noncommunicable diseases and be evermore dependent upon medical/caregiver services as well as lifelong drug prescriptions. We have witnessed decades of medical symptom management for diseases like asthma instead of actual medical cures. The increased disease burden combined with underlying misregulated inflammation among the aged population created the tipping point for this pandemic.

The proposed solution for a system that has resulted in more disease and medical needs rather than more health is also the path toward sustainable healthcare: treating the patient as a multi-species human holobiont and pursuing a microbiome-first medical strategy to support long term health. The biological reality is that the patient is mainly microbial when it comes to genes and gene products affecting metabolism. This paper describes the perspective of human diseases converging rather than diverging (as has been the trend with both medical coding and pharmacological therapies). Importantly, both infectious and noncommunicable diseases can begin with a shift from a healthy, diversified and balanced microbiome to an inflammation-promoting pathobiome. The opportunity to medically manage the patient's microbes permits barriers (gut, airways, skin, urogenital tract) to be protected and human physiology, metabolism, and immune system balance to be positively affected. Metabologenomics and enhanced colonization resistance are presented as microbe management tools that can move us toward a more sustainable approach to healthcare.

Keywords: Human Holobiont; Microbiome, Sustainable healthcare, Pathobiome, Microbiome-first medicine, SARS-CoV-2, Microimmunosome, Pathobiont, Colonization resistance, Metabologenomics
\end{abstract}

Abbreviations: NCDs: Noncommunicable Diseases; WHO: World Health Organization; SARS-CoV-2: severe acute respiratory syndrome coronavirus 2 also (Covid 19); Staph A: Staphyloccocus aureus

\section{Introduction}

\section{Healthcare's Promise}

Twenty first century healthcare has featured two major promises that when combined would move the world significantly toward a sustainable healthcare system. The first promise originated out of the human genome project. It was that medicine must be personalized (tailored to the specific characteristics of the individual patient) and be precise. Individualized strategies to prevent disease and specific medical treatments should be based on the individual patient's genetics, prior medical history and holistic biology [1]. The second promise was to significantly reduce the prevalence of a major category of human diseases: noncommunicable diseases (NCDs). These are chronic diseases such cardiovascular disease, obesity, cancer, chronic respiratory diseases (e.g., asthma), diabetes and major depressive disorders. A clarion call in healthcare went out to stop the incessant rise in NCDs, which are the number one world-wide killer.

The World Health Organization recently reported that noncommunicable diseases (NCDs) accounted for $71 \%$ of all deaths globally [2], and the economic burden of these diseases is alarmingly unsustainable [3]. The prevalence of these diseases has been on the rise and recent medical care approaches have, thus far, failed to stop the increasing global prevalence of these diseases and conditions. One of the insidious features of NCDs is that they often begin in childhood either as fully diagnosed diseases (e.g., childhood asthma, type 1 diabetes, food allergies, neurobehavioral 
disorders, and obesity), or as biomarkers of later-life onset disease (e.g., cardiovascular disease) [4].

Management of these disease conditions has led to an agingrelated progression of additional, and predictably specific comorbid NCDs. Serious chronic diseases are most often medically managed across decades with the target of reducing symptom severity. To date, few NCDs are actually cured. But the symptom management medical priority has actually paved the way for the epidemic of NCD co-morbidities and has resulted in increased disease burden with aging [5]. The problem is that when the core dysbiosis (physiological, metabolic and microbial) that drive an NCD are not corrected, the underlying unbalanced and autodestructive immune responses can produce additional NCDs. Asthma is one example where comorbidities are common and are problematic among existing patients and can include rhinitis, vocal cord dysfunction, gastro-esophageal reflux, psychiatric disorders, obesity and obstructive sleep apnea [6].

Obesity is one of the metabolic syndrome conditions where inflammatory spread in tissues buttressed by immune dysregulation and microbiome dysbiosis can result in a myriad of co-morbid chronic diseases. Over time and with aging of the obese patient cohort, the chronic low-grade inflammation at the core of obesity spreads among tissues and organs and additional co-morbid diseases (e.g., neuropsychiatric) arise [7]. At least 32 different co-morbidities (including 12 different cancers) are more likely among the obese population than the general population [8]. With each new co-morbid NCD that is diagnosed comes additional medical care and potential caregiver requirements as well as the likelihood of decades of polypharmacy. Polypharmacy is not only a problem in developed countries $[9,10]$ but has also emerged as an issue in developing nations [11]. As previously described in 2015 [12], the entire healthcare system has been unable to address the ongoing NCD epidemic for one simple reason: the patient is not a human, the patient is a human holobiont that is mainly microbial.

\section{More Than Human}

The human holobiont (also called the human superorganism) is a composite organism composed of the human mammalian body combined with the human resident microbiome (the bacteria, archaea, viruses, fungi along with their genes) that also inhabit our body at the routes of exposure to the external environment (e.g., gut, airways, skin, urogenital tract). Note that these body locations with rich human microbiota are also the portals of entry for infectious agents. The vast majority of our trillions of microbes are not only friendly to our body, but they provide critical metabolic, physiologic, regulatory and host defense (e.g., colonization resistance) functions that are needed for a healthy life. All higher organisms on earth operate as composites or halobionts [13].
That is a fundamental nature of life on earth (which is primarily a microbial planet). While the development and function of all of our physiological systems are influenced by the human microbiome, the interaction between the microbiome and the immune system is perhaps the most critical in determining the likelihood that an infant's life will be filled with health vs. disease [14]. The immunetissue barrier (gut endothelium lining)-microbiome operates as a systems biology unit that has been termed, the microimmunosome [15].

In a keystone 2017 paper considering the holobiont nature of life on earth, Pitlik and Koren [16] presented a 40,000 ft. view of the states of health vs. disease for multi-species organisms. They argue that probably all diseases of the holobiont (human, plant and animal) including acute, chronic, infectious, noninfectious, local or systemic result from a transformational shift from a beneficial, healthy microbiome to a disease state termed the pathobiome (a dysbiotic microbiome that permits pathogens to gain a foothold which reduces health). In the pathobiome, endogenously or exogenously invading pathobionts (sometimes benign organisms that can become pathogenic under the right circumstances) take advantage of a perturbed ecosystem to transform a healthy microbiome into one that promotes disease. It is this unifying view of human disease in the majority microbial holobiont that should change how we practice medicine and deliver healthcare.

The biological reality of the human holobiont patient means that all prevention and treatment much be directed at the holobiont, not at the human mammal. Greater than $99 \%$ of our genes are microbial via the microbiome with less than one percent human chromosomal $[17,18]$. If we are to move toward sustainable healthcare, our microbial majority can no longer go unseen and forgotten.

\section{Future Healthcare Providers as Microbe Man- agers}

One of the reasons why the human microbiome exerts such a major influence over our immune system has to do with location. For example, the majority of our immune cells are located where they are separated from the gut microbiome by only a one-cell thick epithelial lining [19]. Similar immune-microbiota proximities are found across barriers in skin, airways, and the urogenital tract. Bi-directional communication occurs between the immune system and the human microbiota across these barriers as part of microimmunosome function. For effective immune balance to occur and be maintained (including properly regulated inflammation), the microbiome must also be in ecological balance and the barriers uncompromised. The starting point is the microbiome. Barrier function and immune balance flows from there. Hence, fully personalized and sustainable 21st century healthcare must be 
focused on the human body's main driver of physiological balance, neurological and immunological health, and metabolic potential: our human microbiota. Managing microbes will be a critical path to reach the core cause(s) (e.g., systems biology dysbiosis) that underlie inflammation driven NCDs.

\section{The SARS-Cov-2 (Covid-19) Pandemic Reveals our Misguided Healthcare Focus}

The 2020-21 pandemic of SARS-CoV-2 demonstrated precisely how little has been accomplished in either of these two prioritized areas. The default for SARS-CoV-2 treatment and vaccinations has not featured personalized medicine. In fact, to date it has been much closer to a one-size-fit all public health approach where everyone in the world is considered genetically, physiologically, metabolically, and microbially the same. But that is far from reality.

The SARS-CoV-2 pandemic has revealed the price we must pay for embracing an unsustainable healthcare system that has allowed populations to go through life accumulating ever increasing numbers of NCDs, with a treatment plan desperate to manage more and more symptoms across multiple diseases, but with few if any, actual NCD cures. It is this aging, multi-disease-burdened population that represents the fruits of our medical labors and that was specifically targeted by and most susceptible to death by SARS-CoV-2 [20,21]. With SARS-CoV-2 the danger of severe, life threatening outcomes is linked to the likelihood of cytokine storms [22]. This is a massive inflammatory reaction usually in tissues that can rapidly lead to immune damage of the tissues and loss of function $[23,24]$. It is not surprising that the greatest at-risk population for severe, life threatening SARS-CoV-2 is the higher age group carrying underlying misregulated inflammation associated with NCDs such as metabolic syndrome (diabetes, obesity, cardiovascular disease) $[25,26]$.

Because the core causes of NCDs have not been addressed during symptom management for NCDs, aberrant immune inflammatory responses to SARS-CoV-2 have now created a life-threatening situation among a significant percentage of the population. We need to recognize that patients carrying the burden of NCDs with underlying immune dysfunction including misregulated inflammation are really ticking time bombs. Because the core cause of dysbiosis and disease has yet to be corrected via previous medical treatments, the first environmental trigger requiring an immune response (in this case SARS-CoV-2 infection) could be the last in that patient group. Medical treatments failing to get at root causes of NCDs place patients in significant jeopardy as revealed by the SARS-CoV-2 pandemic.

With SARS-CoV-2, those older individuals who carried comorbid inflammatory-driven chronic diseases and conditions (cardiovascular disease, diabetes, hypertension, obesity, chronic kidney disease) were at the greatest risk for hospitalization [27]. This increased risk for a cytokine storm among the diseaseburdened geriatric population demonstrates a critical lesson in the goal of sustainable healthcare. It is no longer acceptable to treat human inflammation driven disease in such a way that disease symptoms are managed but the underlying cause of the disease (often immune with microbiome dysfunction) is not corrected. Over time and with aging of the population, underlying misregulated inflammation can spread among tissues resulting in additional comorbid diseases. This is one of the additional risks that can arise from childhood-adult obesity [8].

\section{Focal Points in Moving Toward Sustainable Healthcare}

SARS-CoV-2 laid bare our failures in managing the human holobiont health and demonstrated why healthcare must adjust to meet the 21st century reality of human biology. The human microbiome should be a primary medical focus since it is the genetic and metabolic core for both the microimmunosome and human holobiont overall. Then the first question is what does one manage among the trillions of human-dwelling bacteria, archaea, viruses (including bacteriophages), and fungi? Afterall, there are trillions of possibilities ready for adjustment. It is very clear that we need to start practicing microbiome-first medicine and using all the available tools to ensure that ecologically balanced, healthy microbiomes are the standard for both preventative and therapeutic medicine.

\section{Two Recent Findings Can Help Guide our Way Toward Sustainability}

1. Metabologenomic analysis, focusing on microbial genes that affect human metabolism and physiology, can provide important indicators of healthier microbiomes across all stages of human development. This is illustrated in two recent examples.

First, the critical importance of having newborn babies and early infants colonized by Bifidobacterium longum ssp infantes was demonstrated by the fact that this species can imprint immune system development to reduce the risk of autoimmune and allergic diseases [28]. Reduced levels of this bacterial species can also promote enteric inflammation [29]. Additionally, B. infantis and related strains are the best microbial metabolizers of human milk oligosaccharides [30]. This type of analysis demonstrates the importance of a baby's early seeding with specific Bifidobacterial strains and species and can guide pediatric medicine.

Second, a study using microbiota transfer therapy (including fecal transplants) for autism spectrum disorders has shown long term benefits. The desired microbiota transfers were guided by metabolomic goals to correct the ASD associated pathobiome. Two years after the treatments, improvements were seen in both 
gastrointestinal and ASD symptoms as well as in fecal microbiota diversity [31]. Again, metabologenomically-guided alterations in the microbiome are more likely to result in a healthier shift in the microbiome and in overall human physiology.

2. Using microbes to fight microbes is a useful strategy. Optimizing colonization resistance can result in host defense resiliency and reduce the likelihood that pathogens can gain a foothold.

At the heart of this health promoting tool is an understanding of ecological communities within the microbiome. For example, even small genome guided tweaks in gut microbial populations appear to be sufficient to provide significant resistance against pathogens such as Salmonella enterica serovar Typhimurium [32]. In another example, Staphylococcus aureus (Staph A) and infant carriage in the nose microbiome is a risk factor for asthma [33]. But a related Staphylococcus species, S. epidermidis competes with Staph A and metabolites for S. epidermidis can inhibit Staph A biofilm formation [34]. This opens up potential new opportunities to reduce risk by using microbes-to-fight-microbes as a strategy.

\section{Conclusion}

A microbiome-first medical approach is needed if we are to move toward sustainable healthcare. The best defense against potential pathogens like SARS-CoV-2 is a healthy microbiome and a microbiome-driven balanced immune system. The microimmunosome is the body's physical location where health vs. disease and, potentially, life vs. death can be most easily shifted.

The pathobiont theory of disease has gained added support since the long-standing boundary between communicable (infectious) diseases and NCDs appears to be falling away. Evidence suggests that NCDs may be at least contextually communicable $[35,36]$. As a result, these two categories of human disease seem to be more artificial than real. Specific pathogens can take advantage of reduced colonization resistance during microbial dysbiosis, and the emerging pathobionts can promote specific NCDs. In addition to the Staph A-asthma connection, at least some forms of inflammatory bowel disease can be promoted by a specific group of pathogens led by adherent invasive Escherichia coli $[37,38]$. We have met the enemy and it is the pathobiont. The frontline medical solution to protect against disease is likely within us, and it is a relative of the pathobiont.

\section{Acknowledgements}

The author thanks Bill Cullifer, Co-Founder of the World Asthma Foundation, for his helpful discussions on asthma and sustainable healthcare and also thanks Janice Dietert for her editorial suggestions.

\section{Conflict of Interest}

The author declares that there is no conflict of interest

\section{References}

1. Brittain HK, Scott R, Thomas E (2017) The rise of the genome and personalised medicine. Clin Med (Lond) 17(6): 545-551.

2. World Health Organization (2021) Noncommunicable Diseases.

3. Kaminini S (2019) World Economic Forum, Why the $21^{\text {st }}$ century's biggest health challenge is our shared responsibility.

4. Olson M, Chambers M, Shaibi G (2017) Pediatric Markers of Adult Cardiovascular Disease. Curr Pediatr Rev 13(4): 255-259.

5. Dietert RR, De Witt JC, Germolec DR, Zelikoff JT (2010) Breaking patterns of environmentally influenced disease for health risk reduction: immune perspectives. Environ Health Perspect 118(8): 1091-1099.

6. Kaplan A, Szefler SJ, Halpin DMG (2020) Impact of comorbid conditions on asthmatic adults and children. NPJ Prim Care Respir Med 30(1): 36.

7. Castanon N, Lasselin J, Capuron L (2014) Neuropsychiatric comorbidity in obesity: role of inflammatory processes. Front Endocrinol (Lausanne) 5: 74

8. Dietert R (2016) The Human Superorganism How the Microbiome is Revolutionizing the pursuit of a healthy life. Dutton Penguin Random House New York, NY USA, pp. 352.

9. Chou J, Tong M, Brandt NJ (2019) Combating Polypharmacy Through Deprescribing Potentially Inappropriate Medications. Journal of Gerontological Nursing 45(1): 9-15.

10.Zazzara MB, Palmer K, Vetrano DL, Carfi A, Graziano O, et al. (2021) Adverse drug reactions in older adults: a narrative review of the literature. Eur Geriatr Med 12(3): 463-473.

11. Bhagavathula AS, Gebreyohannes EA, Fialova D (2021) Prevalence of Polypharmacy and Risks of Potentially Inappropriate Medication Use in the Older Population in a Developing Country: A Systematic Review and Meta-Analysis. Gerontology 11: 1-10.

12. Dietert RR, Dietert JM (2015) The Microbiome and Sustainable Healthcare. Healthcare (Basel) 3(1): 100-129.

13. Bordenstein SR, Theis KR (2015) Host Biology in Light of the Microbiome: Ten Principles of Holobionts and Hologenomes. PLOS Biol 13(8): e1002226.

14. Dietert R, Dietert J (2012) The Completed Self: An Immunological View of the Human-Microbiome Superorganism and Risk of Chronic Diseases. Entropy 14: 2036-2065.

15. Dietert RR (2017) The microbiome-immune-host defense barrier complex (microimmunosome) and developmental programming of noncommunicable diseases. Reprod Toxicol 68: 49-58.

16. Pitlik SD, Koren O (2017) How holobionts get sick-toward a unifying scheme of disease. Microbiome 5(1): 64

17. Yang X, Xie L, Li Y, Wei C (2009) More than 9,000,000 Unique Genes in Human Gut Bacterial Community: Estimating Gene Numbers Inside a Human Body. PLOS ONE 4(6): e6074.

18. Gilbert JA, Blaser MJ, Caporaso JG, Jansson JK, Lynch SV, et al. (2018) Current understanding of the human microbiome. Nat Med 24(4): 392400 .

19. Ramanan D, Cadwell K (2016) Intrinsic Defense Mechanisms of the Intestinal Epithelium. Cell Host Microbe 19(4): 434-441. 
20. Dean E, Skinner M, Yu HP, Jones AY, Gosselink R, et al. (2021) Why COVID-19 strengthens the case to scale up assault on non-communicable diseases: role of health professionals including physical therapists in mitigating pandemic waves. AIMS Public Health 8(2): 369-375.

21. Pal R, Bhadada SK (2020) COVID-19 and non-communicable diseases. Postgrad Med J 96(1137): 429-430.

22. Yongzhi X (2021) COVID-19-associated cytokine storm syndrome and diagnostic principles: an old and new Issue. Emerg Microbes Infect 10(1): 266-276.

23. Thepmankorn P, Bach J, Lasfar A, Zhao X, Souayah S, et al. (2021) Cytokine storm induced by SARS-CoV-2 infection: The spectrum of its neurological manifestations. Cytokine 138: 155404.

24. Panoskaltsis N (2021) Are all cytokine storms the same? Cancer Immunol Immunother 70(4): 887-892.

25. Di Salvo E, Di Gioacchino M, Tonacci A, Casciaro M, Gangemi S, et al. (2021) Alarmins, COVID-19 and comorbidities. Ann Med 53(1): 777 785.

26. Barone MTU, Ngongo B, Harnik SB, Oliveira LX, Végh D, et al (2021) COVID-19 associated with diabetes and other noncommunicable diseases led to a global health crisis. Diabetes Res Clin Pract 171: 108587.

27. Fried MW, Crawford JM, Mospan AR, Watkins SE, Munoz B, et al (2021) Patient Characteristics and Outcomes of 11721 Patients with Coronavirus Disease 2019 (COVID-19) Hospitalized Across the United States. Clin Infect Dis 72(10): e558-e565.

28. Henrick BM, Rodriguez LR, Lakshnikanth T, Pou C, Henckel E, et al. (2020) Bifidobacteria-mediated immune system imprinting early in life. bioRxiv.

29. Henrick BM, Chew S, Casaburi G, Brown HK, Frese SA, et al. (2019) Colonization by B. infantis EVC001 modulates enteric inflammation in exclusively breastfed infants. Pediatr Res 86(6): 749-757.
30. Lawson MAE, O’Neill IJ, Kujawska M, Gowrinadh Javvadi S, Anisha Wijeyesekera, et al. (2020) Breast milk-derived human milk oligosaccharides promote Bifidobacterium interactions within a single ecosystem. ISME J 14(2): 635-648.

31. Kang DW, Adams JB, Coleman D Pollard EL, Malonado J, Sharon Mc Donough-Means, et al. (2019) Long-term benefit of Microbiota Transfer Therapy on autism symptoms and gut microbiota. Sci Rep 9: 5821.

32. Brugiroux S, Beutler M, Pfann C, Garzetti D, Ruscheweyh HJ, et al. (2016) Genome-guided design of a defined mouse microbiota that confers colonization resistance against Salmonella enterica serovar Typhimurium. Nat Microbiol 2: 16215.

33. Tang HHF, Lang A, Teo SM, Judd LM, Gangnon R, et al. (2021) Developmental patterns in the nasopharyngeal microbiome during infancy are associated with asthma risk. J Allergy Clin Immunol 147(5): 1683-1691.

34. Glatthardt T, Campos JCM, Chamon RC, de Sá Coimbra TF, Rocha GA, et al. (2020) Small Molecules Produced by Commensal Staphylococcus epidermidis Disrupt Formation of Biofilms by Staphylococcus aureus. Appl Environ Microbiol 86(5): e02539-19.

35. Finlay BB, CIFAR Human, the Microbiome (2020) Are noncommunicable diseases communicable? Science 367(6475): 250-251.

36. Huang R, Ju Z, Zhou PK (2020) A gut dysbiotic microbiota-based hypothesis of human-to-human transmission of non-communicable diseases. Sci Total Environ 745: 141030.

37. Nadalian B, Yadegar A, Houri H, Olfatifar M, Shahrokh S, et al. (2021) Prevalence of the pathobiont adherent-invasive Escherichia coli and inflammatory bowel disease: a systematic review and meta-analysis. J Gastroenterol Hepatol 36(4): 852-863

38. Baldelli V, Scaldaferri F, Putignani L, Del Chierico F (2021) The Role of Enterobacteriaceae in Gut Microbiota Dysbiosis in Inflammatory Bowel Diseases. Microorganisms 9(4): 697. 